\title{
Experimental studies of heat transfer between crystal, crucible elements, and surrounding media when growing large-size alkali halide ingots with melt feeding
}

\author{
V. I. Goriletsky, B. V. Grinyov, O. Ts. Sidletskiy, V. V. Vasilyev, \\ M. M. Tymoshenko \& V. I. Sumin. \\ Institute for Scintillation Materials NASU, Kharkiv, Ukraine
}

\begin{abstract}
A series of time stages of heat transfer between the growing crystal and the growth furnace inner elements (different parts of the crucible, water-cooled walls of the growth chamber) have been revealed at growth of AI BVII crystals. Experimental data on temperature changes of these objects have been obtained for the first time using non-contact pyrometry methods.

Some causes of structure defects formation connected with intensification of heat transfer from the growing ingot to the water-cooled walls of the growth chamber and leading to unavoidable melt supercooling have been determined. The causes of crystallization front shape changes initiated by thermal flux from the crucible elements to the melt surface have been established.

Keywords: crystal growth, condensate, heat transfer, temperature distribution, pyrometry.
\end{abstract}

\section{Introduction}

Until now, in comparison to the Czochralsky growth, a lack of direct experimental studies concerning heat transfer processes inside the vacuum water cooled growth camera has been observed for automated continuous feed method developed by Eidelman et al. [1]. In many instances, this fact is connected with the condensate (layer of evaporated melt components) precipitating on growing crystal and growth furnace inner elements. Utilization of IR-pyrometry is, probably, the one suitable method of measurements at the growth of alkali halide 
crystals in vacuum, when the crucible and growing ingot continuously rotate. So, a procedure of direct non-contact pyrometric temperature measurements of crystal surface and crucible elements at different growth stages has been developed by Sidletskiy et al [2].
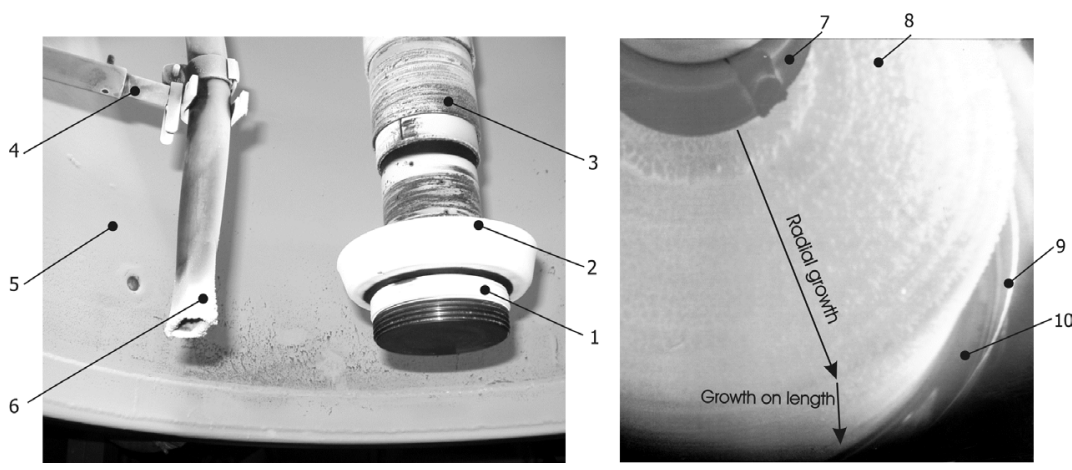

Figure 1: General view of the growth furnace inner surface and the surface of the $500 \mathrm{~mm}$ dia. $\mathrm{NaI}(\mathrm{Tl})$ growing crystal. 1 - lower part of the water cooled crystal holder; 2 - condensate collector; 3 - telescopic protective shield of the water-cooled crystal holder rod; 4 - transport tube holder; 5 - inner surface of the furnace case; 6 - transport tube; 7 - coupling nut of the seed; 8 - upper butt of the growing crystal; 9 - vertical wall of the crucible; 10 - melt. The left photo is made after the unloading of the crystal; the right one is made directly during the growth process.

A growth furnace of "ROST" type setup consists of two essential parts. A thermal camera with crucible is placed in the lower part, and growing crystal are situated in the upper part. Thus, this construction predetermines the direction of heat flux from the heaters through the crucible, melt, and crystal to the water-cooled case of the furnace upper part. A free melt surface, not barred by the crystal, always exists during the crystal growth on a seed. The square of this surface, other conditions being equal, determines the quantity of the precipitating condensate. This fact is of great importance for the growth of scintillation crystals such as $\mathrm{NaI}(\mathrm{Tl}), \mathrm{CsI}(\mathrm{Tl})$, where the dopant is a volatile substance. Following these considerations, cooled inner walls of the growth furnace are the preferable place for condensate precipitation. However, condensate is also observed on the more heated surfaces, as one can see in fig. 1, where the general view of the growth furnace inner surface and growing ingot are presented.

The role of the condensate seems weighty, because it is an insulator that substantially influences the heat transfer in the system. Non-uniformity of condensate thickness makes heat transfer dynamics even more difficult. 


\section{Peculiarities of heat and mass transfer at radial growth and body growth}

The three more important stages of the large single crystal growth with melt feeding can be emphasized, fig. 2: $\mathrm{a}$ - radial (cone) growth; $\mathrm{b}$ - growth on height (body growth) till the moment when crystal upper butt leaves the crucible limits; $\mathrm{c}$ - growth on height after the moment when crystal upper butt leaves the crucible. Let us consider step-by-step the peculiarities of heat exchange between the crystal, crucible elements, inner atmosphere, and furnace case.

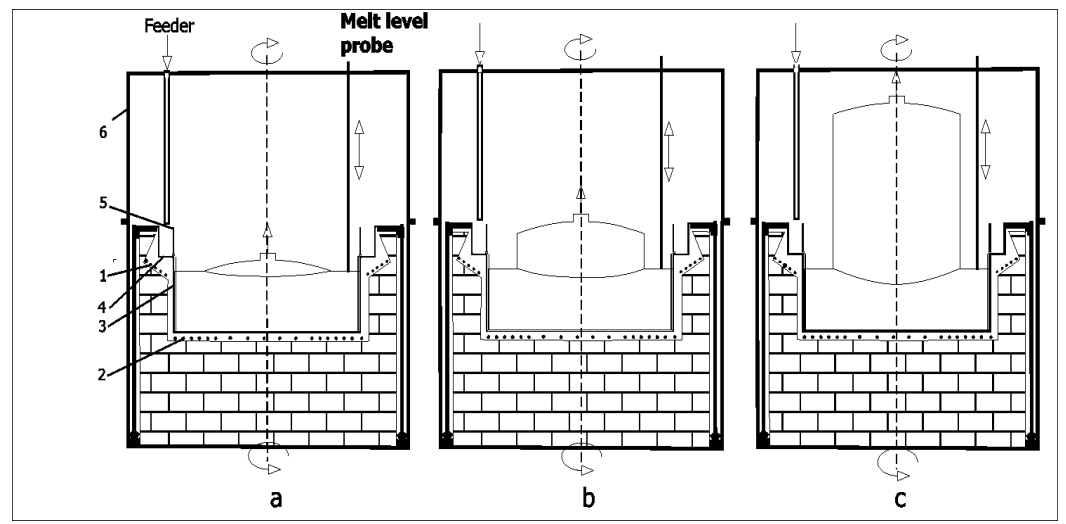

Figure 2: Scheme of the large scintillation single crystal growth with melt feeding: 1 -side heater; 2 -bottom heater; 3 -crucible; 4 - periphery circular vessel (PCV) of the crucible; 5 - upper edge of the crucible vertical wall; 6 - water-cooled case of the furnace.

In accordance with the experimental data, at radial growth till the preset diameter, the crystal is covered with a condensate layer, and its thickness is proportional to the growth time. The exception is a small area near the seed where condensate is absent. The upper butt temperature at this stage, fig. 3, smoothly decreases with time; it is lower in the crystal centre and higher near the butt edge.

So, at the diameter $270 \mathrm{~mm}$, temperature in this area decreases down to $105^{\circ} \mathrm{C}$ in comparison to the crystallization point $\left(621^{\circ} \mathrm{C}\right)$. One can see in, fig. 3 , that in this area the condensate layer is the thickest (under illumination the condensate is lighter, and without illumination it is darker) providing an insulation of this crystal part from radiation passing from the heaters, through the melt and crystal (the latter are high transparent in IR band).

The character of heat exchange on the radial growth stage is seen in fig. 4, where common dynamics of the following parameters are presented at the different growth stages: 
- $\quad$ bottom heater temperature $\left(t_{b o t}\right)$ providing automated temperature correction by signals from the melt level probe (see fig. 2);

- $\quad$ program controlled side heater temperature $\left(t_{\text {side }}\right)$;

- $\quad$ temperature of the PCV bottom $\left(t_{p e r}\right)$ measured by the pyrometer;

- temperature of the upper edge of the crucible wall $\left(t_{\text {wall }}\right)$ measured by the pyrometer.

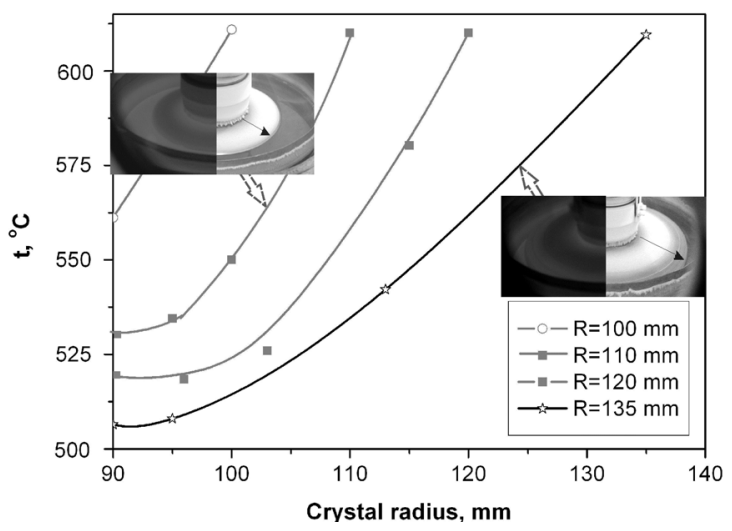

Figure 3: Temperature distribution at the upper butt of $\operatorname{CsI}(\mathrm{Na})$ crystal for current values of crystal radius 100, 110, 120, and $135 \mathrm{~mm}$. Above and below are photos of growing crystals with additional illumination (left parts of the photos) and without it (right parts of the photos).

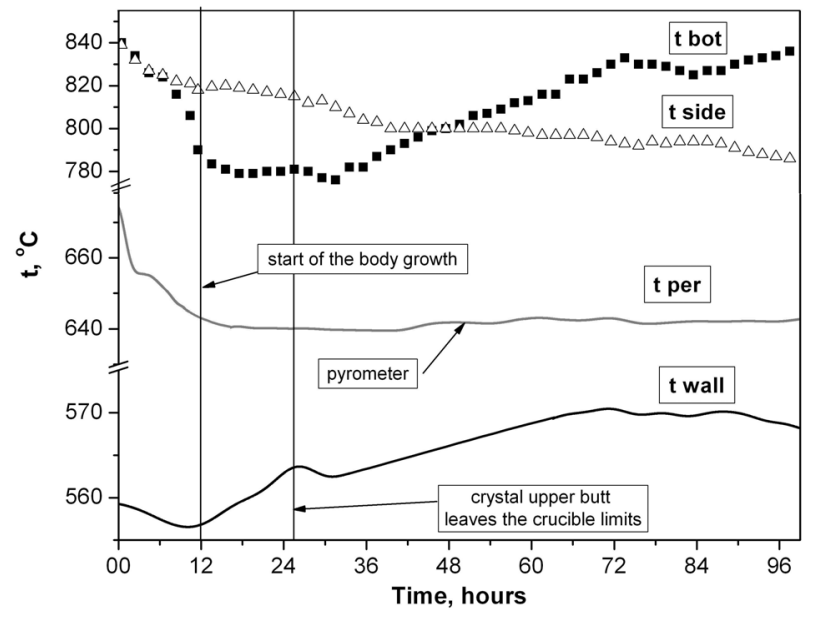

Figure 4: Dynamics of $t_{\text {bot }}, t_{\text {side }}, t_{\text {per }}$ and $t_{\text {wall }}$ at growth of $300 \mathrm{~mm}$ dia. CsI(Na) crystal. The three stages of growth are separated by vertical lines. 
One can see from fig. 4 that at this growth stage initiated by decrease of $t_{b o t}$ and $t_{\text {side }}$, the decrease of $t_{\text {per }}$ and $t_{\text {wall }}$ is also observed. This fact indicates the presence of radial temperature gradient in melt taking part in formation of a convex crystallization front (CF). Herein, it is obvious that the crucible elements above melt (see fig. 2, stage a) partly suppress a heat transfer in the direction from the crystal to the water cooled case. Such role of the crucible elements in common heat exchange is proportional to the distance from the melt surface to the crucible upper edge $\left(H_{\text {free }}\right)$. At growth with melt feeding, $H_{\text {free }}$ remains constant during all the process, and this parameter is not investigated in the present work.

The next stage of the growth - body growth when all the crystal is still inside the crucible, fig. $2 \mathrm{~b}-$ is characterized by the start of $t_{\text {wall }}$ increase and stabilization of $t_{\text {per }}$ with a simultaneous continued decrease of the control parameters $t_{\text {side }}$ and $t_{b o t}$ (see fig. 4). The cause of the crucible vertical wall temperature increase can be described unambiguously: heat emitting from the crystal side surface. Here we must note that a part of the crystal side surface near the melt surface is not covered with condensate and is capable to transmit a significant part of radiant heat flux.

Moreover, at the start of constant diameter crystal growth, the square of the emitting surface substantially increases proportionally to the speed of pulling of the ingot from melt. Thus, if the vertical crucible wall temperature in the cone growth stage is determined, in general, by the $t_{\text {side }}$ parameter which must be decreased for radial growth, $t_{\text {wall }}$ increases proportionally to crystal cylindrical part length $H_{c y l}$ (see fig. 4) on stage b. On the other hand, with $H_{c y l}$ increase, the crystal migrates towards the crucible wall upper edge where the influence of the cooled furnace walls increases.

The picture of heat transfer presented is confirmed by the measurements of temperature changes on the crystal upper butt, fig. 5. Analyzing them, the following conclusions can be made:

- the axial temperature gradient in crystal decreases due to increase of $H_{c y l}$ and heat removal from the side ingot surface;

- the representative minimum at the temperature distribution curve at $H_{c y l}=$ $5-15 \mathrm{~mm}$, hereinafter, slowly transforms into the plateau at $\mathrm{R}=100-135 \mathrm{~mm}$ (fig. $5, H_{c y l}=235 \mathrm{~mm}$ ) due to the increase of the temperature gradient in the crystal.

Now considering the third growth stage, let us look back again to figs. $2 \mathrm{c}$ and 4 . The main difference of this stage from the previous one is that the crystal (i.e. its upper part) is already situated outside the crucible limits stimulating even more powerful heat removal from the ingot. As a result, the melt overcools, and an automated system provides the sign reversal of the $d t_{b o t} / d H_{c y l}$ derivative. Then the growth process acquires the more stationary character.

As the conclusion of this chapter, it should be noted that the slower $t_{b o t}$ increase, fig. 4 , and the decrease of the axial temperature gradient at $H_{c y l}$ above $200 \mathrm{~mm}$, fig. 5 inclusion, can indicate changes in heat removal conditions from the growing crystal to the water cooled furnace walls. The authors plan further studies of heat and mass transfer process in this direction. 


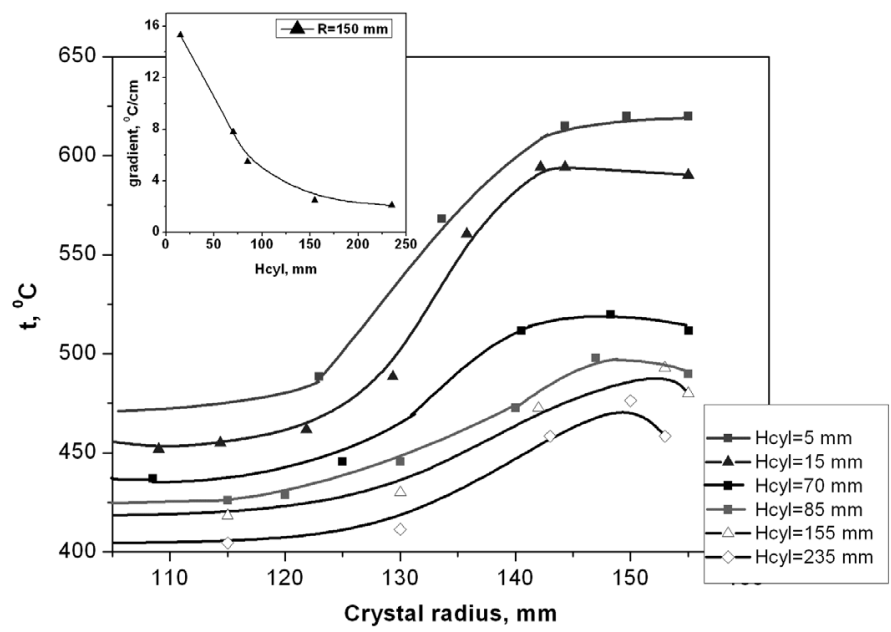

Figure 5: Temperature distribution on the $\mathrm{CsI}(\mathrm{Na})$ crystal upper edge at cylindrical part lengths $H_{c y l}=0,15,70,85,155,235 \mathrm{~mm}$. In addition, the corresponding changes of axial temperature gradient on the crystal upper butt (on $150 \mathrm{~mm}$ radius) are shown.

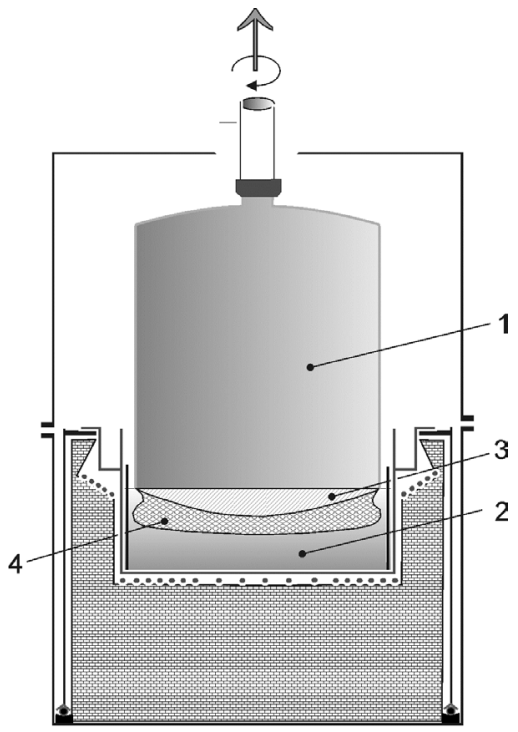

Figure 6: Scheme of CF transformation at crystal growth with additional $t_{\text {side }}$ correction and without it: 1 - growing crystal; 2 - melt; $3-\mathrm{CF}$ with $t_{\text {side }}$ correction; $4-\mathrm{CF}$ without the correction. 


\section{Heat and mass transfer and control above the properties of the growing crystal}

Substantial changes in CF volume at crystal elongation are observed experimentally, fig. 6 , at growth of crystals with extremely large diameter (the ratio of crystal diameter to crucible diameter $D_{\text {crys }} / D_{\text {cruc }}>0.83$ ).

Herein, melt level falls, and this reflects in the corresponding increase of the dopant concentration in the crystal with $H_{c y l}$ increasing in accordance with Goriletsky et al. [3]. Close proximity of the crystal side surface to the crucible vertical wall, as shown above, increases the temperature in the PCV, which is accompanied, in turn, by the increase of volatile dopant evaporation and leads to changes in thermal conditions. So, when $H_{c y l}$ increases, $t_{p e r}$ must be continuously controlled by $t_{\text {side }}$ temperature in order to stabilize the thermal conditions in an area near CF. For this reason, the additional contour of automated control system (ACS) of PCV bottom temperature $\left(t_{p e r}\right)$ was developed. Referring to fig. 4 , we can see the results of such control: at constant $t_{\text {per }}$ (its stabilization starts at body growth stage) the $t_{\text {side }}$ parameter continuously decreases, thus, compensating heat input from the crystal to the PCV.

As mentioned above, heat exchange substantially increases on the stage $c$, and ACS must react on this by adequate increase of the $t_{b o t}$ parameter. Macrodefects represented by the foreign phase inclusions, or dendritic structures that situated along the $\mathrm{CF}$, fig. 7, sometimes can be found just in this part of the grown ingots (at $H_{c y l}=40-60 \mathrm{~mm}$ ).

The represented local defects are situated along the CF shape and in the areas where crystal diameter increase is observed. This effect is accompanied by an increased rate of crystallization above the preset value.

Thus, insufficient sensitivity of the ACS to changes in general heat exchange in the growth furnace is one more probable cause of the macrodefects formation.

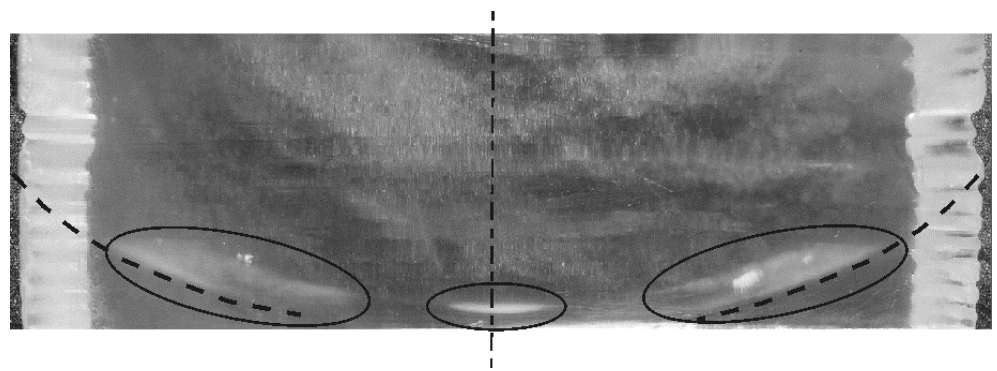

Figure 7: $\quad$ Macrodefects (denoted by the ellipses) along the CF (dashed lines) found on the longitudinal cut of $\operatorname{CsI}(\mathrm{Na})$ single crystal. 


\section{Conclusions}

1. Three time phases of heat transfer corresponding to the three general stages of crystal growth are established:

1.1. Thermal field limiting the heat removal from the crystal is formed on the radial growth stage. Primary, it is formed due to condensate layer precipitation, then, due to the close proximity of the crucible PCV creating temperature "barrier". This phase is accompanied by decrease of the control $\left(t_{\text {side }}, t_{b o t}\right)$ and measured $\left(t_{\text {wall }}, t_{\text {per }}\right)$ parameters.

1.2. The moment of initial body growth coincides with the start of heat removal from the side crystal surface where condensate layer has not yet formed. This stage fixes the start of $t_{\text {wall }}$ increase under stable $t_{\text {side }}$ and decreasing $t_{b o t}$.

1.3. More active heat transfer between the crystal, crucible walls, and water cooled case starts when conic part of crystal leaves the crucible limits. This fact is confirmed by the continuing $t_{\text {wall }}$ increase and increase of $t_{b o t}$ by the ACS. During all the body growth stage, the condensate does not precipitate on the conic part and on the area of 5-10 mm width near melt. Its thickness smoothly increases at $10-20 \mathrm{~mm} H_{c y l}$ height, and, further, condensate is distributed uniformly.

2. The main cause of dopant concentration fluctuations in single crystals of extreme diameter and different composition is the $\mathrm{CF}$ volume changes caused by the absence of $t_{\text {side }}$ correction. The effect is manifested in changes of thermal conditions near the $\mathrm{CF}$ as a result of heat exchange between crystal cylindrical part and PCV.

3. The increase of heat transfer from the growing crystal to the surrounding media, and inadequate ACS reaction, lead to the formation of local short-term throws of foreign phase admixtures at the CF due to melt overcooling and a corresponding increase of local mass rate of crystallization. The evidence of this effect is the crystal diameter increase.

\section{References}

[1] Eidelman, L.G., Goriletsky, V.I., Protsenko, V.G. et al, Automated pulling from the melt - an effective method of growing large alkali-halide crystals for optical and scintillation applications. J. Crystal Growth, 128, p. 1059, 1993.

[2] Sidletskiy, O.Ts., Goriletsky, V.I., Grinyov, B.V., Sumin, V.I., Sizov, O.V., Tymoshenko, M.M., Monitoring of thermal fields on surface of alkali halide single crystals grown from the melt. Functional Materials, 12(4), p. 591, 2005.

[3] Goriletsky, V.I., Grinyov, B.V., Sumin, V.I., Tymoshenko, M.M., Changes in crystallization conditions when growing large single crystals at melt feeding. Functional Materials, 11, p. 806, 2004. 